\title{
Schematic models for fragmentation of brittle solids in one and two dimensions
}

\author{
F.P.M. dos Santos, R. Donangelo, and S.R. Souza \\ Instituto de Física, Universidade Federal do Rio de Janeiro, Caixa Postal 68528, \\ 21941-972 Rio de Janeiro - RJ, Brazil
}

\begin{abstract}
Stochastic models for the development of cracks in 1 and 2 dimensional objects are presented. In one dimension, we focus on particular scenarios for interacting and non-interacting fragments during the breakup process. For two dimensional objects, we consider only non-interacting fragments, but analyze isotropic and anisotropic development of fissures. Analytical results are given for many observables. Power-law size distributions are predicted for some of the fragmentation pictures considered.
\end{abstract}

Key words: Fracture, size distributions, power-law

PACS: 89.75.-k, 05.90.+m, 46.50.+a

\section{Introduction}

The breakup of a system into many pieces, i.e. fragmentation, is a subject of intensive investigation in different areas of science and engineering $[1,2,3,4]$. The interest in this phenonemon ranges from studies of the observed size distributions of atomic nuclei [2,3] and chains of molecules [4] to asteroids [5], ice floes $[6,7,8]$, brittle solids [9,10,11,12,13], thin glass plates[14], eggshells $[15,16]$, frozen potatoes [17], fluids [13], drops in general $[18,19]$, etc. The size distributions observed in the breakup of these systems exhibit, in general, a power-law behavior, suggesting, in some cases, at least, that the process is related to critical phenomena.

This peculiarity has led to the development of many schematic models which, to a large extent, disregard microscopic details of the fragmenting macroscopic objects. The fact that the size distributions seem to be fairly insensitive to the constituent elements of the fragmenting body [17] gives strong support to this approach. Therefore, different treatments, based on quite general assumptions, 
have been proposed to explain the characteristics of the fragmentation of brittle solids. These approximations include, for instance, mean-field treatments $[20,21,22,23]$, fractal analysis [14,24], dynamical models of granular solids [25], random forces stopping models [26], and schematic branching-merging models $[27,28]$ (for a recente review see [1] and references therein).

In this work, we present schematic models for the breakup of rods and flat brittle objects. For the latter case, our models are intended to describe the fragmentation of objects which suffer a strong impact on one of its sides, in contrast to those proposed in $[20,21,22,23,27]$, in which the stress is uniformly distributed inside the body. Effects associated with anisotropy are also investigated. In the one-dimensional case, we compare the properties of the fragments produced when the interaction between them can be neglected, i.e. when they are ejected from the parent body, with the case in which they continue interacting during the breakup process.

In sect. 2 we present the models and make analytical predictions related to the size distributions, whereas in sect. 3 we discuss and interpret the results. Conclusions are drawn in sect. 4.

\section{The models}

The main characteristic of the models presented below is that they can be viewed as stochastic processes, in which strict mass conservation is imposed in each event. We present below their detailed formulation.

\subsection{1-dimensional models}

Objects whose lengths are large compared with their cross-sections are represented by one-dimensional segments of a line. Thus, in this subsection we consider fractures on a line of unitary length. The number of fractures $N$ is related to the violence of the impact on the object. More specifically, $N$ points $\left\{x_{i}\right\}, i=1 \ldots N$, are associated with the fractures and two adjacents points delimit a fragment, so that $N+1$ fragments are formed at the end of the process.

We concentrate on two different fragmentation models, which aim at describing distinct breakup scenarios:

i- Non-Interacting Fragments (NIF1), where fracture points are sequentially generated and the $(i+1)$-th crack can only appear at the right hand side of the $i$-th fissure. It is numerically implemented by selecting the first random 
point $x_{1}$ uniformly in the interval $(0,1)$. Then, the next crack point $x_{2}$ is uniformly chosen in the interval $\left(x_{1}, 1\right)$, and so on. This corresponds to a simplified picture for the breakup of an object when the impact zone is concentrated close to its left edge, similar to the (almost) perpendicular fall of a rod. In this case, the dynamics cannot produce further fractures in fragments that have already been released from the parent fragment.

ii- Interacting Fragments (IF1), in which the broken pieces exert stress on the others, leading to further cracks. For simplicity, at the $i$-th step, one of the $i+1$ fragments is chosen with equal probability and a fissure point is sampled uniformly inside the selected fragment.

The analytical construction of the NIF1 model can be achieved through the following considerations:

1- When the $i$-th crack point is made, there exists an equivalence between the fragment just formed and the remaining of the parent body.

2- Let us assume that, after the $i$-th fracture, the parent body has length $l$. Then, as the $(i+1)$-th fissure is produced, the probability of it having length $\chi$ must be inversely proportional to its parent's length $l$.

Upon denoting by $P_{i}(\chi)$ the probability density of creating a fragment of size $0<\chi<1$ at the $i$-th crack, one may then write:

$$
P_{i+1}(\chi)=\int_{\chi}^{1} \frac{1}{l} P_{i}(l) d l
$$

By noticing that $P_{1}(\chi)=1$, this recurrence relation can be iterated, leading to:

$$
P_{i}(\chi)=\frac{[-\log (\chi)]^{i-1}}{(i-1) !}
$$

so that the probability density of observing a fragment of size $\chi$, produced at any stage of the fragmentation process is:

$$
P(\chi)=\frac{1}{N} \sum_{i=1}^{N} P_{i}(\chi)=\frac{1}{N} \sum_{i=1}^{N} \frac{[-\log (\chi)]^{i-1}}{(i-1) !} .
$$

For $N$ large, the above expression can then be approximated by:

$$
P(\chi) \approx \frac{1}{N} \exp [-\log (\chi)]=\frac{1}{N} \chi^{-1}
$$

It is worth mentioning that normalization is apparently lost in this approx- 
imation, since the integral of $1 / \chi$ from 0 to 1 diverges. However, the above expression is strictly valid only for $N \rightarrow \infty$ and, therefore, the $1 / N$ factor would cancel out the divergency. Thus, this expression should be considered as an approximation for finite (but large) $N$. Nevertheless, the results shown in the next section reveal that this formula is fairly accurate for modest values of $N$.

One should notice that these results are qualitative different from those corresponding to uniform fragmentation [27], which should take place when the object suffers a violent impact equally distributed over its length. In this case, the probability density of finding a fragment of size $\chi$, after $N$ fractures is [27]:

$$
P_{N}(\chi)=N(1-\chi)^{N-1}
$$

The analysis of the IF1 model is more involved. Although we could not obtain closed expressions, we found useful recurrence relations which allow one to compute the properties of the model very easily. First, one should notice that when a fragment is cracked, the probability distribution for the size of the generated fragment is equal to that of the remnant. Then, the probability density of having a fragment of size $\chi$ after the first crack is made, $\Psi_{1}(\chi)$, can be expressed as:

$$
\Psi_{1}(\chi)=\frac{1}{2}\left[P_{1}(\chi)+P_{1}(\chi)\right]
$$

where $P_{i}(\chi)$ is given by Eq. (2). The normalization factor $1 / 2$ is associated with the number of fragments formed after the first crack of the system.

When the second fracture is made, there are two contributions which should be taken into account:

a) a fragment of size $\chi$ can be produced by the crack of a fragment of size $l$, $\chi<l<1$, or

b) the cracking of a selected fragment may lead to a piece of any size, but the unbroken fragment has size $\chi$.

Since (a) can also lead to a fragment of size $1-\chi$ and a remaining part of size $\chi$, which are statistically equivalent, one may write:

$$
\Psi_{2}(\chi)=\frac{1}{3}\left[2 \int_{\chi}^{1} \frac{1}{l} \Psi_{1}(l) d l+(2-1) \Psi_{1}(\chi)\right]
$$

where the first term accounts for the contribution of (a) and the second one 
is associated with (b). The factor $(2-1)$ accounts for the degeneracy of the remaining fragments in (b), recalling their statistical equivalence. The above expression can be further expanded through the use of Eqs. (1) and (6), and one obtains:

$$
\Psi_{2}(\chi)=\frac{1}{6}\left[4 P_{2}(\chi)+2 P_{1}(\chi)\right]
$$

The general expression for $\Psi_{i}(\chi)$ may be obtained, iteratively, through:

$$
\Psi_{i}(\chi)=\frac{1}{i+1}\left[2\left[\int_{\chi}^{1} \frac{1}{l} \Psi_{(i-1)}(l) d l\right]+(i-1) \Psi_{(i-1)}(\chi)\right] .
$$

More especifically, if $\left\{a_{i}^{(k)}\right\}$ denote the coefficients of the expansion of $\Psi_{i}(\chi)$ at the $i$-th step:

$$
\Psi_{i}(\chi)=\sum_{k=1}^{i} a_{i}^{(k)} P_{k}(\chi)
$$

the above recurrence relation gives:

$$
a_{i}^{(k)}=\frac{1}{i+1}\left[2\left(1-\delta_{k, 1}\right) a_{(i-1)}^{(k-1)}+(i-1) a_{(i-1)}^{(k)}\right],
$$

where Eq. (1) has been used and $\delta_{i, j}$ is the usual Kronecker Delta function. Starting from $a_{1}^{(1)}=1$ and $a_{1}^{(k)}=0,1<k \leq N$, one may thus generate all the coefficients associated with the $N$-th fracture of the system. This procedure is extremely fast and requires a very small amount of computational effort.

\subsection{2-dimensional models}

The 2-dimensional objects considered in this sub-section are squares of unitary sides. We assume that, during the fracture process, the broken pieces detach from the body and do not fragment afterwards. The models we discuss aim at describing some of the gross features of the fragmentation of a square plate caused by a strong impact on one of its sides. Therefore, straight fissures always start at the left edge of the square and end up at one of its borders or at another crack, whichever is found first.

As in the 1-dimensional case, the number of fractures $N$ is associated with the strength of the impact on the object. The position of each starting point of a 
crack is uniformly selected along the left edge of the object. We consider two different pictures for the propagation of the cracks:

a) Non-Interacting Isotropic Fissure (NIIF2): in which a crack grows as a straight line with equal probability along any direction inside the square.

b) Non-Interacting Anisotropic Fissure (NIAF2): where a straight line representing a crack propagates only along selected directions. This version of the model aims at describing the fragmentation of objects, such as cristaline solids, whose internal structure leads to preferential cracking directions. For the sake of simplicity, only two possible directions are considered, $-\pi / 4$ or $+\pi / 4$, with respect to the perpendicular to the left edge of the square. Each of these two directions is chosen with equal probability.

These scenarios lead to qualitatively different fragmentation patterns. This is illustrated in Fig. 1, which shows samples of the patterns obtained for different values of $N$ for the two cases we have considered.

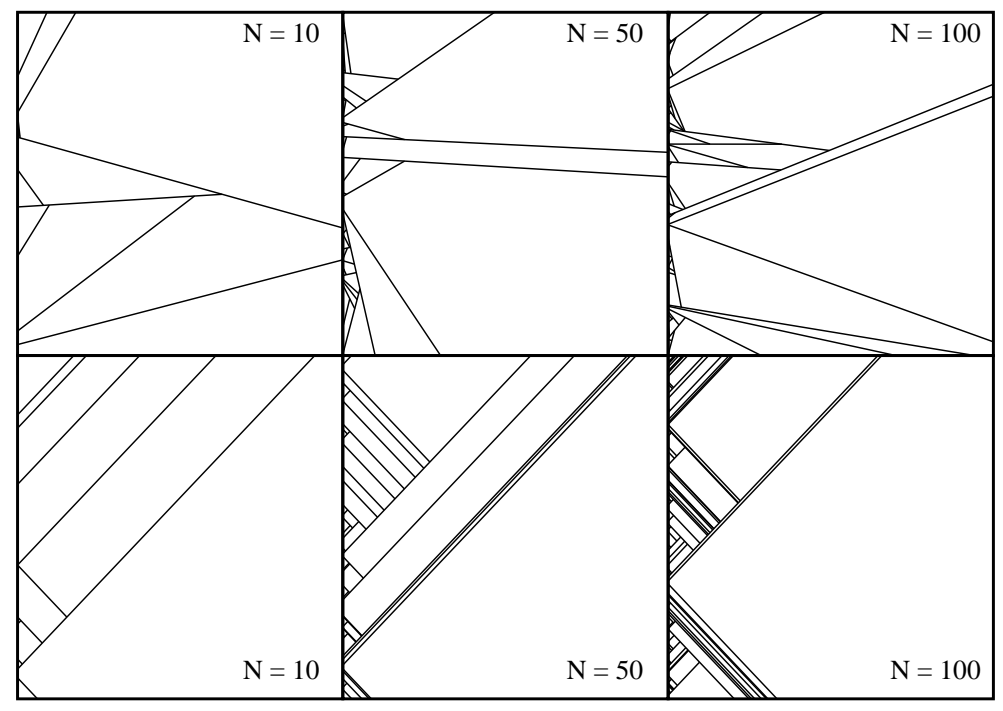

Fig. 1. Fragmentation patterns produced by the isotropic (upper panel) and anisotropic (lower panel) propagation of the fractures, for $N=10, N=50$, and $N=100$ cracks.

In contrast to the 1-dimensional cases, analytical predictions are much more difficult in the present situation. However, particular considerations can be made for very small areas. Among the complex geometric objects that may be formed, triangled shaped pieces are much more likely to contribute in this area region, at least for $N$ not too large. The area of the triangles is $\chi^{2} / 2$, where $\chi$ denotes the size of their bases. Since the distances between two adjacent cracks $\chi$ are statistically distributed according to Eq. (5), the probability density $P_{N}(A)$ of observing a fragment of area $A$, if the system undergoes $N$ fractures, is given by: 


$$
\begin{aligned}
P_{N}(A) & =\int_{0}^{1} \delta\left(A-\frac{\chi^{2}}{2}\right) N(1-\chi)^{N-1} d \chi \\
& =\frac{N}{\sqrt{2 A}}[1-\sqrt{2 A}]^{N-1}, A<<1 .
\end{aligned}
$$

In this expression, we have neglected contributions from other geometric shapes.

Similar considerations also hold for the NIAF2 model, but thin trapezoidal shapes, such as those which appear in Fig. 1, also must be considered. However, the corresponding area should also be proportional to $\chi^{2}$. Therefore, the area distribution, for small values of $A$, should also be a power-law with exponent $-1 / 2$, and a similar behavior is expected to be observed in both models.

\section{Results}

Computational experiments have been carried out for all the models described in the previous section. The corresponding results are presented below, together with their interpretations, accompanied by the analytical explanations we have obtained.

\subsection{1-dimensional models}

The probability density of observing a fragment of size $\chi$, when the violence of the impact on it is such that $N$ fractures are made on the system, has been predicted in the last section, for the two 1-dimensional scenarios we considered. In Fig. 2, we compare our predictions to the results obtained in our computer simulations.

It shows that the size distributions corresponding to the different fragmentation modes are qualitatively different. Particularly, one notices that the interaction among the fragments leads to smaller fragment sizes, compared to the NIF1 model. This is quite reasonable, on physical grounds, since any further interaction, after their formation, would reduce their average size, compared to the case in which they do not interact. A striking feature of the NIF1 model is that the approximation, given by Eq. (4), holds for 10 decades, for $N$ as small as $N=50$, as anticipated in the last section. As a matter of fact, the analytical predictions reproduce the data to a great degree of accuracy.

The difference between the two fragmentation scenarios may be further illustrated by considering the average size of the fragments generated at the $i$-th 


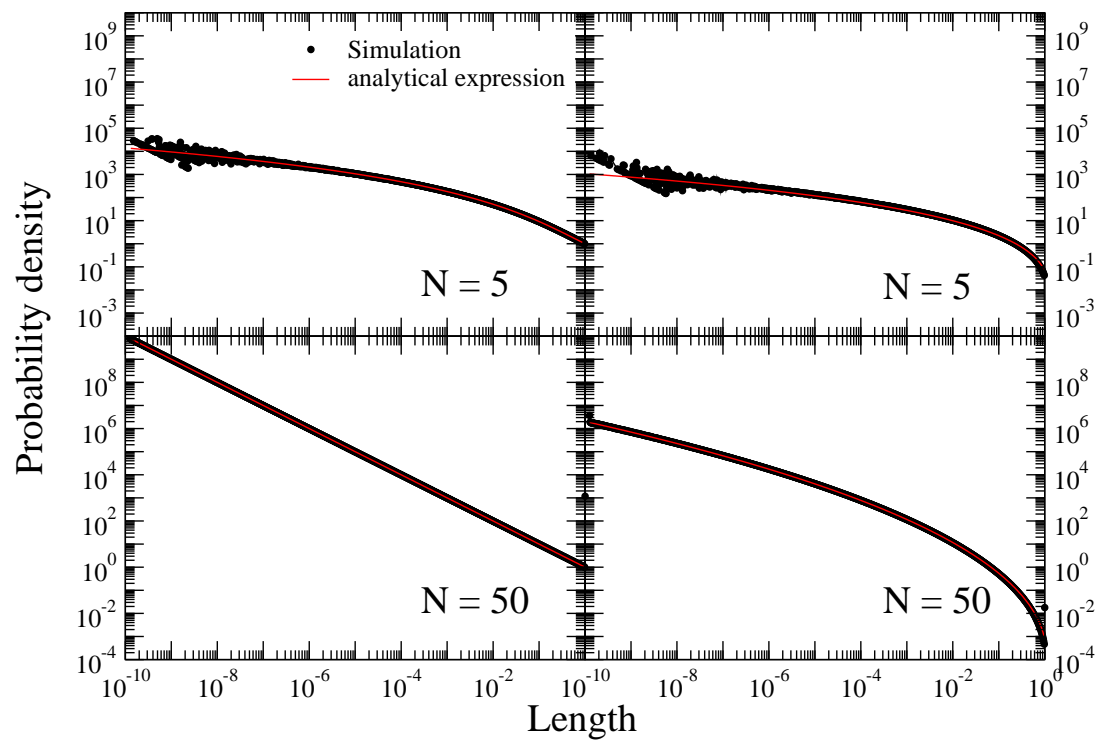

Fig. 2. (Color online) Simulation of 1-dimensional models compared to the analytical predictions of Eqs. (3) and (9). Results corresponding to the NIF1 model are shown at the left panel, whereas those associated with the IF1 are displayed on the right panel.

step. In the NIF1 model, the average size of the fragment created at the $i$-th step is given by:

$$
\left\langle\chi_{i}\right\rangle=\int_{0}^{1} \chi P_{i}(\chi) \mathrm{d} \chi=\frac{1}{2^{i}} .
$$

To arrive at this expression, we have used Eq. (2).

If the fragments are allowed to interact, as in the case of the IF1 model, the average size can be estimated in the following way. The $i$-th fissure is made with equal probability on each of the $i$, already existent, fragments. The average size of these fragments is equal to $1 / i$. Therefore, the size of the $(i+1)-t h$ fragment, created at the $i$-th step, corresponds to:

$$
\left\langle\chi_{i}\right\rangle=\frac{1}{2 i} .
$$

These results show that the average size of the fragments produced at a given step in the IF1 model is, in general, much larger than in the case of the NIF1 model. This prediction is illustrated in Fig. 3, as well as the corresponding simulation data. The agreement between the analytical formulae and the numerical simulations is, once again, remarkably good.

This conclusion apparently contradicts that drawn by the analysis of the size distributions displayed in Fig. 2, i.e. that the interacting fragment picture gives 


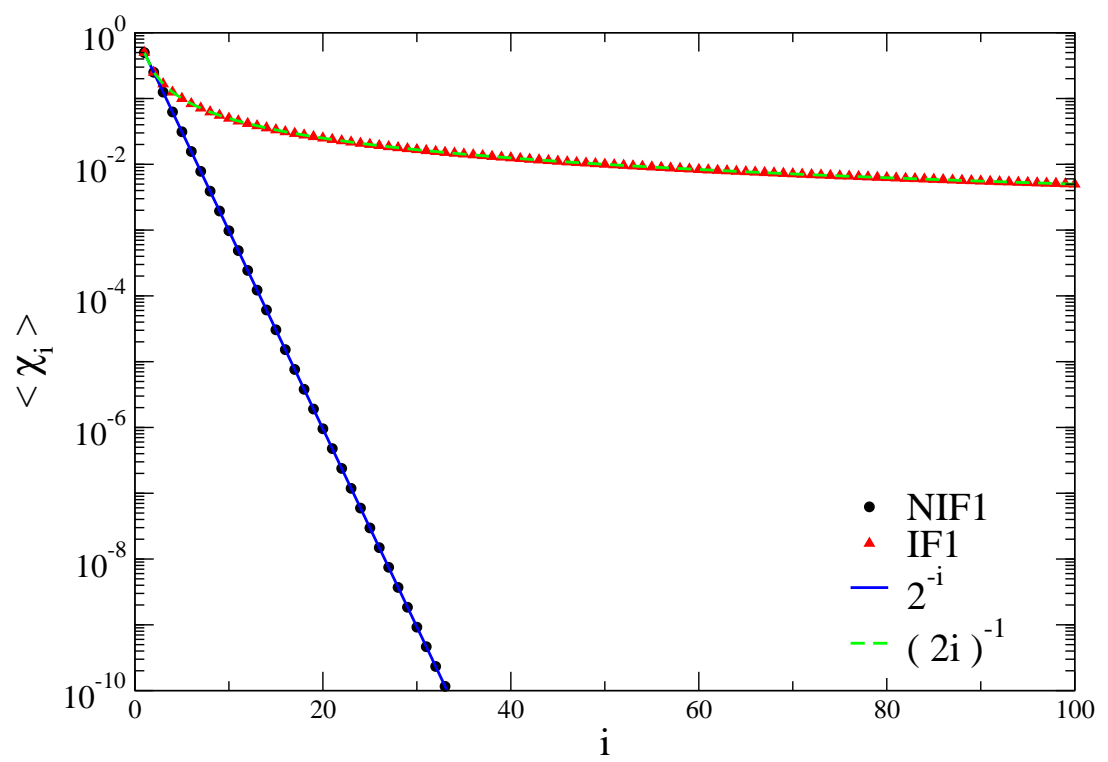

Fig. 3. (Color online) Comparison between the average size of the fragments produced at the $i$-th step in the 1-dimensional models.

much smaller fragments than in the non-interacting scenario. Nevertheless, the apparent inconsistency disappears if one realizes that the survival probability of the fragment produced in the NIF1 model is 1, whereas the fragments are not preserved during the breakup process in the IF1 model. Therefore, large fragments formed at any step in the NIF1 model remain during the whole process, in contrast to those produced in the IF1 model.

The characteristics of the two models may be better understood by analizing the Dalitz plot associated with their final size distributions. This is constructed by selecting the 3 largest fragments within each event, and calculating:

$$
x_{k}=\chi_{k} / \sum_{k=1}^{3} \chi_{k}, \quad(k=1,2, \text { and } 3)
$$

which represent the perpendicular distances to the $k$-th side of an equilateral triangle. In this equation, $\chi_{k}$ corresponds to the size of one of the selected fragments. Thus, a point associated with a given event is plotted inside the triangle using the above expression. To eliminate artificial structures, the indices $\{k\}$ in Eq. (15) are randomized in each event.

By construction, all the points must lie inside the triangle. When the size distribution is dominated by a large fragment, whereas the others are much smaller, one should observe bumps close to the vertices. On the other hand, if the 3 largest fragments have approximately the same size, the peak of the distribution is found near the center of the triangle. Finally, if the size of two of the selected fragments is similar, but much larger than that of the third one, bumps close to the middle point of the triangle sides should be observed. 

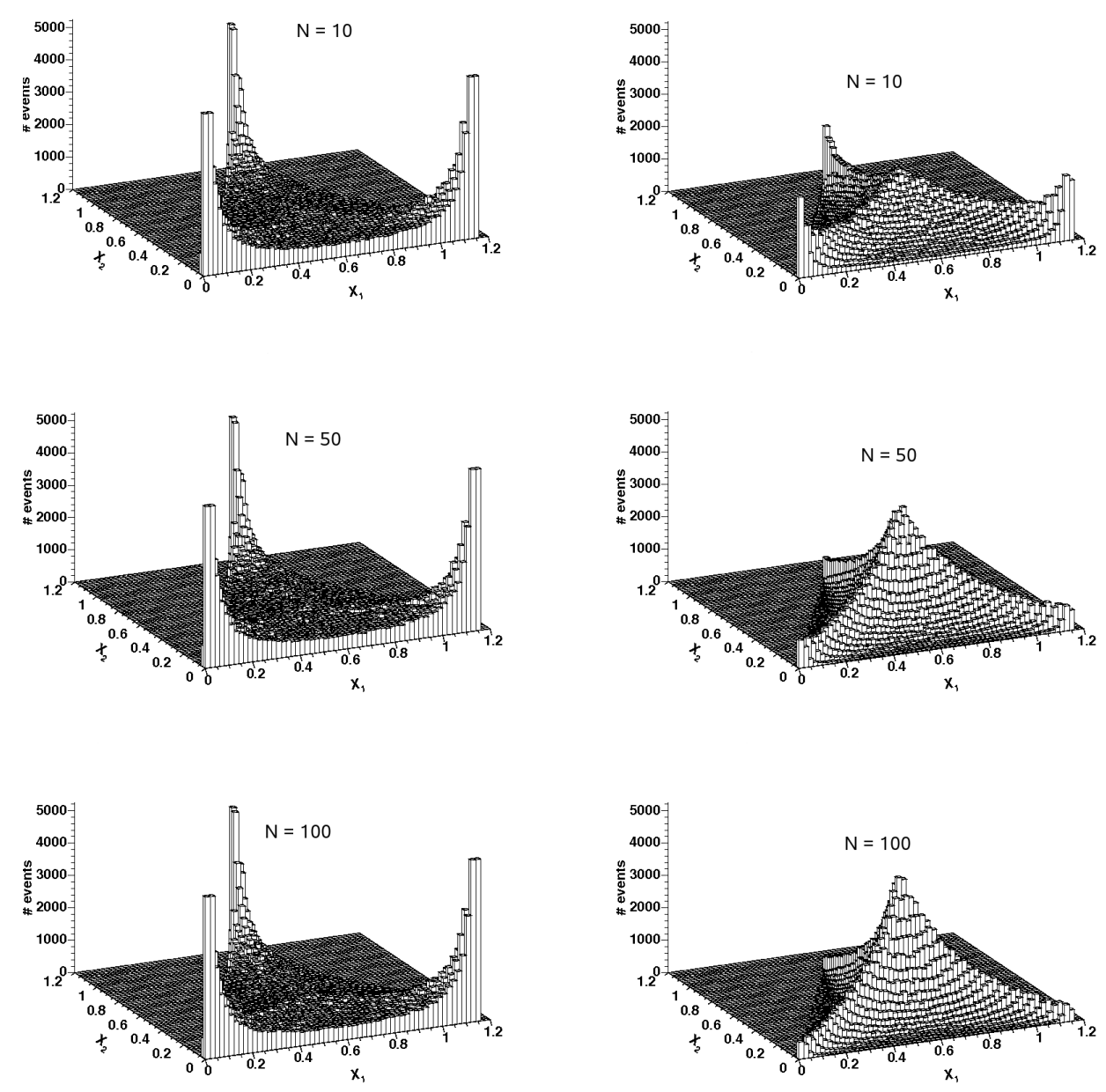

Fig. 4. Dalitz plots associated with the size distributions of the NIF1 (left panels) and IF1 (right panels) models. For details, see text.

The results shown in Fig. 4 reveal that a large fragment is always surounded by smaller ones in the NIF1 model, in contrast to the IF1 model in which the three largest fragments have approximately the same size. Thus, the two fragmentation pictures lead to qualitatively different size distributions.

\subsection{2-dimensional models}

Computer simulations have also been carried out for the fragmentation of square objects, described in sect. 2.2. The results associated with the NIIF2 model are depicted in Fig. 5, for different values of $N$. It is clear that the area distribution exhibits two power-law regimes. One of them has been already predicted in the last section, corresponding to $A^{-1 / 2}$, and is represented by the full lines in this figure. The agreement with the analytical prediction is very good in all cases. However, one notices that deviations appear as $N$ increases. 
This behavior should be expectedi since, as already mentioned, when more cracks are made on the system, the contribution of objects more complex than triangles becomes non-negligible for small areas.

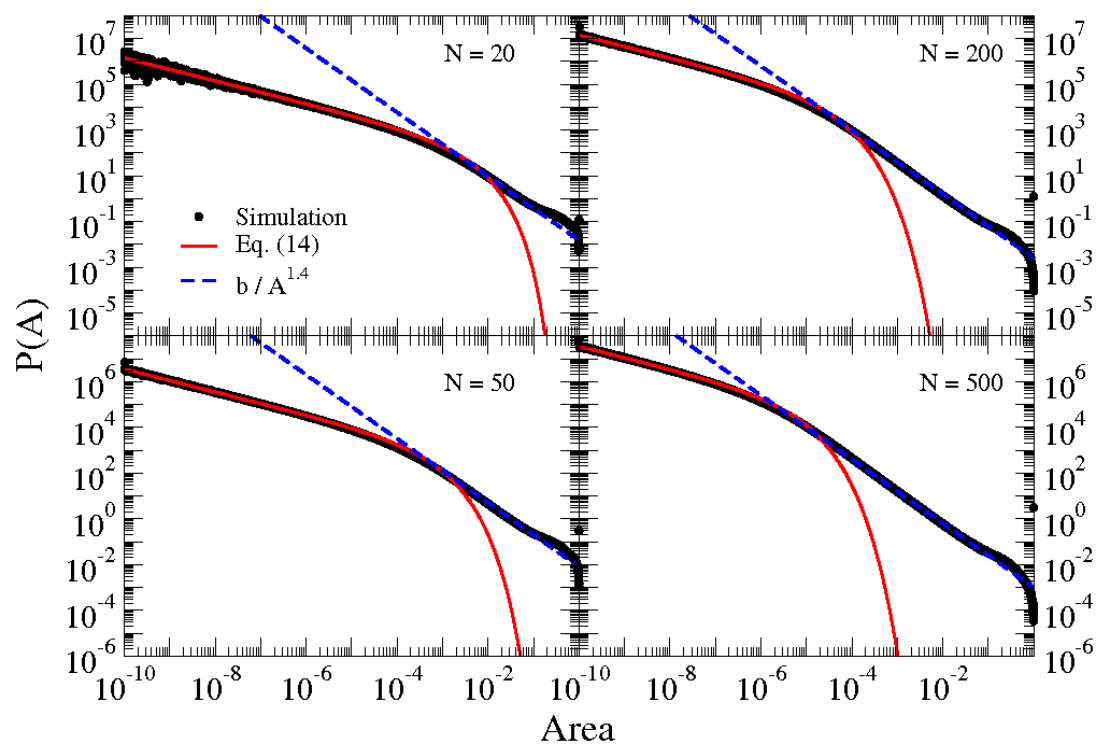

Fig. 5. (Color online) Probability density of observing a fragment of area $A$, obtained with the NIIF2 model for different values of $N$. For details, see text.

One may also notice that another power-law regime, of expoment approximately equal to -1.4 , appears after the breakdown of Eq. (12), in the area regions where no particular geometric shape should dominate the distribution. This is illustrated by the dashed lines in this figure. Since we could not obtain any analytical explanation for this power-law behavior, this is an empirical finding. Nevertheless, it agrees qualitatively with the fractal analysis made in ref. [24] as well as with experimental studies [17] on the fragmentation of rectangular objects, which have demonstrated that the fragment mass distribution (of relatively large pieces) obeys a power-law with exponent approximately equal to $1.15-1.20$. However, those experiments focused on impacts equally distributed over the surface of the plate. Since our model is intended to describe a different picture, the small discrepancy between our results and the experimental observations is quite reasonable.

The qualitative difference between our results and those just mentioned is the existence of two power-law regimes in our model, whereas only one has been observed in the experiments. Unfortunately, it is rather difficult to investigate our prediction experimentally since the second power-law regime should appear for very small values of the area.

The behavior observed in the anisotropic model (NIAF2) is qualitatetively different from the former case, as is illustrated in Fig. 6. The power-law regime, with exponent $-1 / 2$, predicted in the last section is indeed observed, but the area distribution tends to develop a gap for $1 / 3<A<1 / 2$, whereas the 


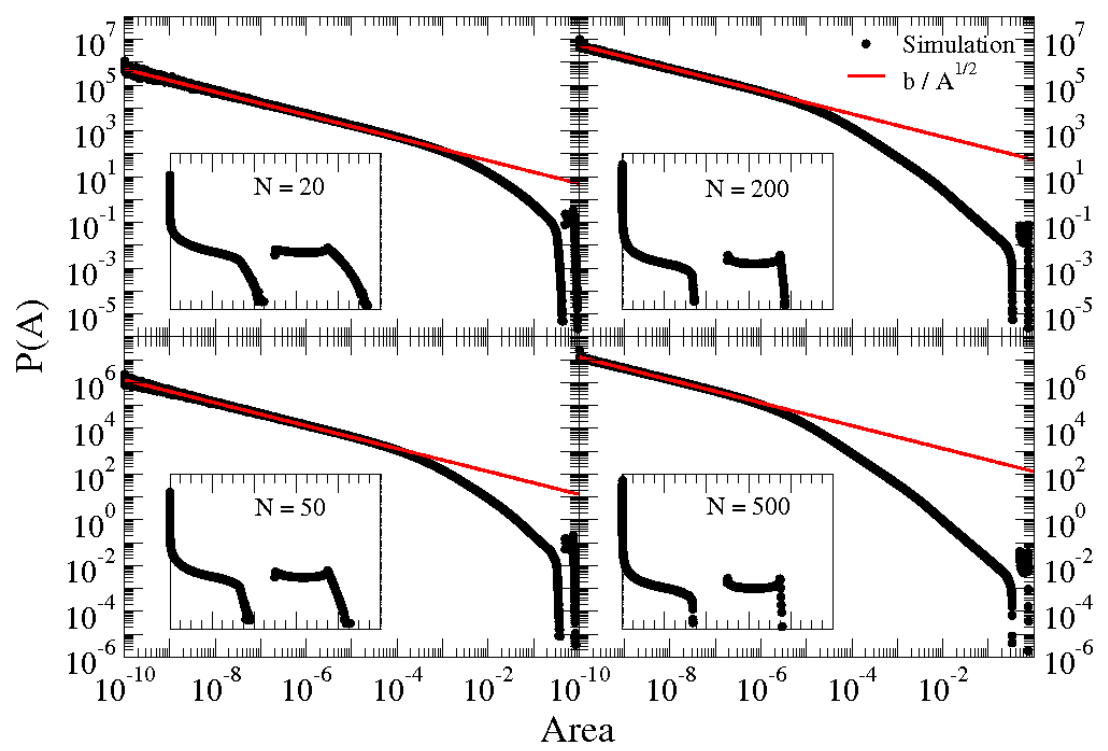

Fig. 6. (Color online) Probability density of observing a fragment of area $A$, obtained with the NIAF2 model for different values of $N$. The inserts correspond to log-linear plots of the respective area distributions. For details, see text.

production of fragments whose $A>0.75$ is hindered. This trend becomes more and more pronounced as $N$ increases.

This feature of the area distribution has a simple explanation, based on the geometric constraints imposed by the anisotropy. In the limit of large $N$, only a few particular shapes may contribute to large areas. More specifically, only those corresponding to the hatched areas in Fig. 7 would not undergo further fractures and survive until the end of the process.
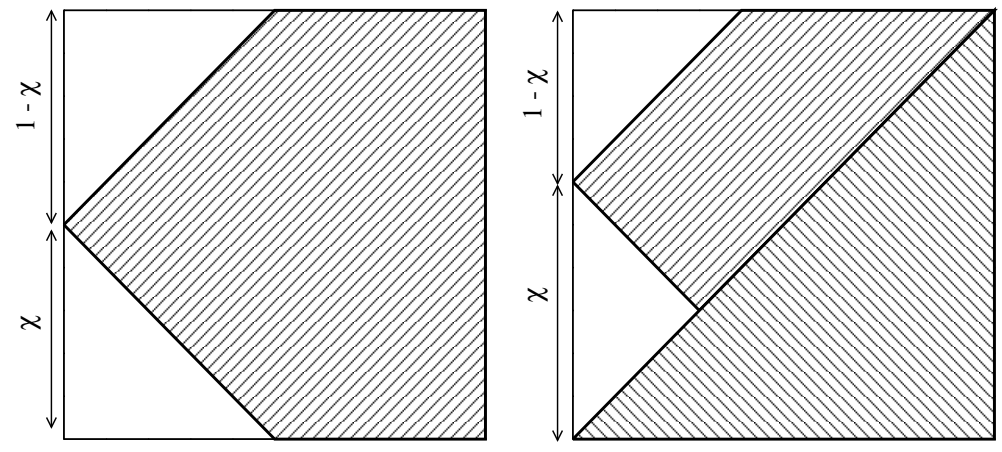

Fig. 7. Fragments of large areas that could be observed in the end of the process for $N$ large.

The area corresponding to the pentagon shown in the left panel of this figure is:

$$
A(\chi)=1-\frac{\chi^{2}}{2}-\frac{(1-\chi)^{2}}{2}
$$


whereas that associated with the trapezoid displayed in the right panel reads:

$$
A(\chi)=\frac{1}{2}-\frac{\chi^{2}}{4}-\frac{(1-\chi)^{2}}{2}
$$

The area of the former lies between $1 / 2 \leq A \leq 3 / 4$, whereas for the latter one has $0 \leq A \leq 1 / 3$. The only big triangle which would not be cut into pieces for large $N$ is the one shown in the right panel, but contributes to the area distribution at the right hand side of the gap. Other shapes have vanishing small survival probability, for large $N$, and would, therefore, be broken into pieces, contributing only to small areas. Thus, the preferred cracking directions give rise to the peculiarities observed in the area distributions. We have checked that qualitatively similar results are also obtained for other cracking directions.

In a more realistic model, fractures should develop preferentially along the selected directions but, with lower probability, other directions should also be allowed. However, one still finds fingerprints on the area distributions. This is illustrated in Fig. 8, where the area distributions for different values of $N$ are shown, in the case where one allows 3 equiprobable cracking directions: $\theta=-\frac{\pi}{4}, \theta=0$, and $\theta=+\frac{\pi}{4}$. Although the gap disappears, due to fissures corresponding to $\theta=0$, the first derivative of $P(A)$ shows a singularity at $A=1 / 2$. One should also notice that, for large areas, but $A<1 / 2$, the distribution follows the same power-law found in the isotropic case.

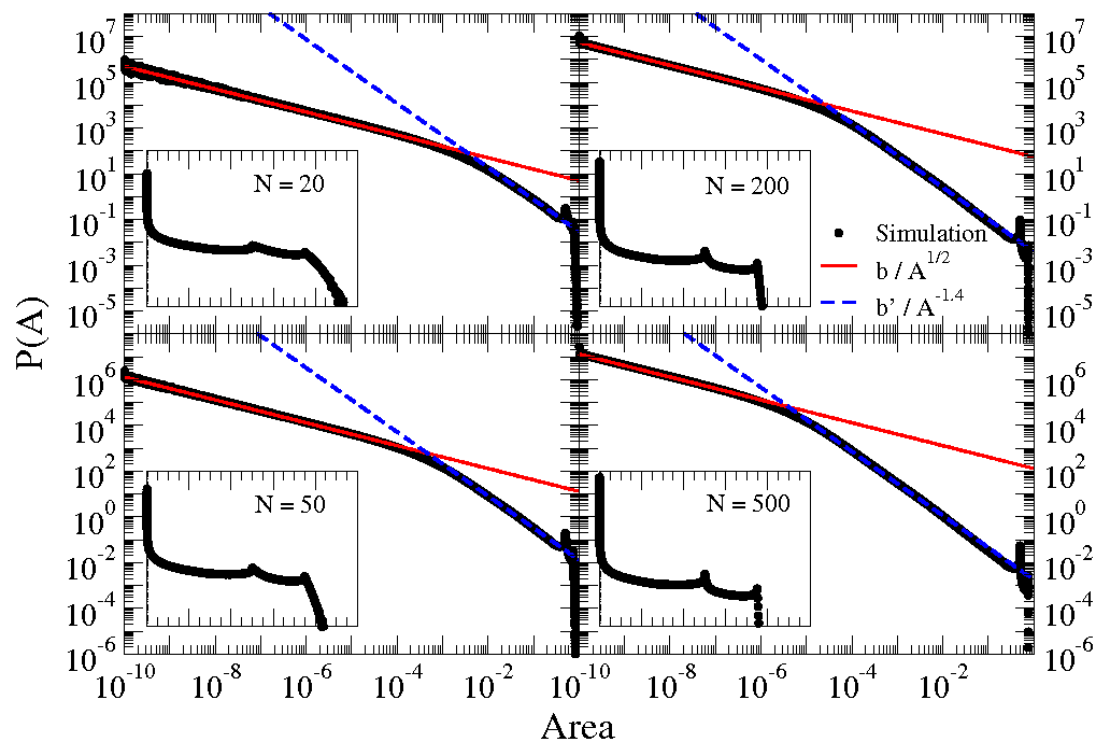

Fig. 8. (Color online) Probability density of observing a fragment of area $A$, obtained with the NIAF2 model for different values of $N$, in the case that 3 cracking directions are allowed. The inserts correspond to log-linear plots of the respective area distributions. For details, see text. 


\section{Concluding remarks}

The schematic models developed in this work for the fragmenation of one dimensional objects revealed that qualitatively different size distributions should be observed whether the fragments interact or not during the breakup process. Size distributions obeying a power-law are only obtained in the noninteracting scenario. Intuitively, this finding is quite reasonable since the interaction among the fragments should lead to the appearance of a scale.

The area distributions, for not too small areas, predicted by our isotropic models are in reasonable agreement with those predicted in [24] and obtained experimentally in [17]. However, from simple considerations, we predict that the probability density of finding fragments of small areas should be a powerlaw with exponent $1 / 2$, in both isotropic and anisotropic fragmenting pictures. Owing to the difficulties in measuring small fragments, we are not aware of experimental observations in this area region, which could be confronted with our predictions.

Our results also suggest that clear signatures of anisotropy effects should be found in the area distributions, for not too small values of $A$. Therefore, we suggest that the fragmentation of anisotropic materials be studied to provide more insight in this phenomenon.

\section{Acknowledgements}

We would like to thank Dr. K. Sneppen, Dr. P.M.C. de Oliveira, and Dr. C.E. Aguiar for many fruitful discussions. We would like to acknowledge CNPq, FAPERJ, and PRONEX(CNPq-FAPERJ) under contract 26.171.176.2003, for partial finantial support.

\section{References}

[1] J. Fineberg, M. Marder, Phys. Rep. 313 (1999) 1.

[2] J. P. Bondorf, A. S. Botvina, A. S. Iljnov, I. N. Mishustin, K. Sneppen, Phys. Rep. 257 (1995) 133.

[3] D. H. E. Gross, Phys. Rep. 279 (1997) 119.

[4] E. E. B. Campbell, F. Rohmund, Rep. Prog. Phys. 63 (2000) 1061.

[5] E. V. Ryan, Annu. Rev. Earth Planet. Sci. 28 (2000) 367.

[6] R. Korsnes, S. Souza, R. Donangelo, A. Hansen, M. Paczuski, K. Sneppen, Physica A 331 (2004) 291. 
[7] A. Chmel, V. N. Smirnov, M. P. Astakhov, Physica A 357 (2005) 556.

[8] A. Chmel, V. N. Smirnov, M. P. Astakhov, J. Stat. Mech. (2005) P02002.

[9] J. J. Gilvarry, J. Appl. Phys. 32 (1961) 391.

[10] J. J. Gilvarry, B. H. Bergstrom, J. Appl. Phys. 32 (1961) 400.

[11] J. A. Aström, R. P. Linna, J. Timonen, P. F. Moller, L. Oddershede, Phys. Rev. E 70 (2004) 026104.

[12] J. Aström, M. Kellomäki, J. Timonen, Phys. Rev. E 55 (1997) 4757.

[13] J. Aström, B. L. Holian, J. Timonen, Phys. Rev. Lett. 84 (2000) 3061.

[14] T. Kadono, M. Arakawa, Phys. Rev. E 65 (2002) 035107.

[15] F. K. Wittel, F. Kun, H. J. Hermann, B. H. Kröplin, Phys. Rev. E.

[16] F. K. Wittel, H. J. Hermann, B. H. Kröplin, Phys. Rev. Lett.

[17] L. Oddershede, P. Dimon, J. Bohr, Phys. Rev. Lett. 71 (1993) 3107.

[18] P. M. C. de Oliveira, T. J. P. Penna, A. R. Lima, J. S. S. Martins, C. Moukarzel, C. A. F. Leite, Cond-mat/0101184.

[19] P. M. C. de Oliveira, C. A. F. Leite, C. V. Chianca, J. S. Martins, C. F. Moukarzel, preprint submitted to Physica A.

[20] G. J. Rodgers, M. K. Hassan, Physica A 233 (1996) 19.

[21] E. Ben-Naim, P. L. Krapivsky, Physica D 107 (1996) 156.

[22] E. Ben-Naim, P. L. Krapivsky, Phys. Lett. A 275 (2000) 48.

[23] E. Ben-Naim, P. L. Krapivsky, Phys. Rev. E 50 (1994) 3502.

[24] G. Huber, M. H. Jensen, K. Sneppen, Fractals 3 (1995) 525.

[25] F. Kun, H. J. Hermann, Phys. Rev. E 59 (1999) 2624.

[26] G. Hernandez, Physica A 323 (2003) 1.

[27] D. Grady, M. Kipp, J. Appl. Phys. 58 (1985) 1210.

[28] R. P. Linna, J. A. Aströn, J. Timonen, Phys. Rev. E 72 (2005) 015601. 